\title{
El derecho de coacción como garantía del equilibrio del derecho
}

Héctor Arrese Igor

\section{(2) OpenEdition \\ Journals}

Edición electrónica

URL: http://journals.openedition.org/ref/320

DOI: $10.4000 /$ ref.320

ISSN: 2258-014X

Editor

EuroPhilosophie Editions

Referencia electrónica

Héctor Arrese Igor, «El derecho de coacción como garantía del equilibrio del derecho », Revista de Estud(i)os sobre Fichte [En línea], 1 | 2010, Publicado el 14 junio 2010, consultado el 10 diciembre 2020. URL : http://journals.openedition.org/ref/320 ; DOI : https://doi.org/10.4000/ref.320

Este documento fue generado automáticamente el 10 diciembre 2020.

(c) EuroPhilosophie 


\title{
El derecho de coacción como garantía del equilibrio del derecho
}

\author{
Héctor Arrese Igor
}

\section{Introducción}

1 Fichte defendió la Revolución Francesa en sus escritos anónimos Reivindicación de la libertad de pensamiento (1793) y Contribución a la rectificación del juicio del público sobre la Revolución Francesa (1794). En estos textos abogó por un Estado que garantice a los ciudadanos las condiciones para el desarrollo de la moralidad, entendida como una forma de auto-legislación. Entre estas condiciones se cuentan la libertad de conciencia y de expresión, indispensables para intercambiar argumentos y dar razones de los motivos que cada uno tiene para actuar. Luego, sin embargo, durante sus años en Jena en los que emprendió la tarea de concebir una filosofía del derecho dentro del sistema de su filosofía, la doctrina de la ciencia, Fichte debió enfrentar el desafío de diseñar un Estado en el que puedan vivir también aquellos individuos cuyo interés no sea el de contribuir al progreso moral de la humanidad.

2 La concepción de un tal estado es, sin duda alguna, uno de los problemas principales en su Fundamento del derecho natural (GNR). ${ }^{1}$ En este texto Fichte revisa los presupuestos de sus primeros escritos e intenta elaborar una teoría del derecho que sea independiente de la ética. Para ello recurre al supuesto motivacional del egoísmo universal, que implica la concentración de los poderes legislativo, judicial y ejecutivo en este último, a fin de concebir un poder tal, cuya realidad efectiva (Wirklichkeit) pueda evitar que cada ciudadano persiga su interés particular y, de este modo, atente contra la estabilidad de la comunidad política. Por consiguiente ya casi no queda lugar en la idea fichteana del Estado para el ejercicio de la soberanía popular, lo cual significa un claro alejamiento respecto de los ideales de la Revolución Francesa, con los que Fichte había simpatizado en un primer momento a comienzos de la misma.

3 Se ha discutido mucho el recurso de Fichte al supuesto del egoísmo universal, criticado especialmente por Hegel en su trabajo sobre el derecho natural de 1802/03. ${ }^{2}$ Pero la 
idea de Estado que Fichte propone en su GNR no puede comprenderse cabalmente, si no se atiende a su fundamentación del derecho de coacción, dado que la idea misma de comunidad legal gira en torno a este eje. En este trabajo me propongo reconstruir la argumentación esgrimida por Fichte para determinar las ideas de un equilibrio del derecho y de un derecho de coacción, entendiendo éste como una mediación indispensable de aquél. De este modo intento hacer un aporte para la comprensión de un texto que ocupa un lugar estratégico en la filosofía política de Fichte.

\section{Deducción del concepto de equilibrio del derecho (Gleichgewicht des Rechts)}

4 Fichte comienza su argumentación en torno a la idea de Estado tomando en consideración la estructura misma del derecho de coacción, porque es el eje del diseño institucional que tiene en mente. La primera cuestión a resolver para fundamentar el derecho de coacción es, por consiguiente, determinar cuándo se ha producido una transgresión al principio del derecho.

5 Si un determinado uso de la libertad es ilegal, entonces debe haber un uso legal de la misma respecto del cual la persona se ha apartado. Es decir, debe haber ciertos límites para establecer el uso legal de la libertad, los cuales deben estar determinados por el principio del derecho. El principio del derecho prescribe una delimitación mutua de esferas iguales de libertad entre las personas o, en palabras de Fichte, un "equilibrio del derecho". (GA I, 3, 410 GNR) La pregunta aquí es, entonces, qué cantidad de libertad corresponde a cada individuo según el principio del derecho. El problema de la cantidad de libertad que cada individuo debe permitirse y permitir al otro tiene sentido sólo si, por un lado, el individuo tiene experiencia de un otro como de un ser que existe realmente fuera de él y limita su propia libertad a través del reconocimiento de la posibilidad de la libertad de ese otro, y, por otro lado, si ese otro limita su libertad del mismo modo.

6 Esta cuestión no podría plantearse, por consiguiente, si se partiera de un sujeto que existe solo y aislado de los demás:

"Si, como sucede en la deducción del derecho originario, se piensa una persona [como existiendo] aislada en el mundo sensible, entonces tiene [esta persona], en tanto y en cuanto no conozca a ninguna persona fuera de ella, el derecho de extender su libertad tanto como pueda y quiera, $y$, si le place, de tomar en posesión para sí todo el mundo sensible. Su [sc. de esta persona] derecho es infinito en su efectividad real (suponiendo que el derecho originario pueda ser un derecho efectivamente real), pues la condición, bajo la cual el mismo tendría que ser limitado, caduca." (GA I, 3, 412 GNR) ${ }^{3}$

7 Ahora bien, la persona aparece en primer lugar en su representación sensible en un cuerpo articulado. El cuerpo de la otra persona ocupa un lugar en el espacio y limita los movimientos de mi persona, al menos en la medida en que mi cuerpo no puede ocupar el lugar que ocupa el cuerpo de la otra persona sin ejercer violencia contra él. Si la otra persona aparece por medio de su cuerpo articulado, entonces la primera deberá limitar los movimientos de su cuerpo para posibilitar a su vez los movimientos del cuerpo de la otra persona. Cada uno podrá limitar la libertad de su cuerpo sólo si el otro también lo hace. 
8 En un primer momento, en el que nadie ha limitado aún su libertad, cada persona está necesariamente en un estado de incertidumbre respecto de la intención de las demás de respetar o no la libertad de los demás. Todas las personas carecen, entonces, en realidad de la información relevante para establecer una relación jurídica. Aun en el caso de que uno supiera que el otro está dispuesto a respetar su derecho originario y decidiera, en consecuencia, permitir que esa otra persona ejerza su influencia sobre aquellos objetos dados en el mundo sensible que en cada caso necesite para realizar sus fines, ninguno podría decidir cuáles de estos objetos deberían ser dejados al arbitrio del otro, dado que nadie estaría en condiciones de saber cuáles son los fines que el otro se propone realizar. Los fines de cada persona sólo se dan en la conciencia de cada uno y, por esto, no se dejan exteriorizar en su totalidad por medio del cuerpo del individuo en cuestión. (cf. GA I, 3, 413-414 GNR)

9 En este estado de incertidumbre nadie puede saber cuáles objetos son propiedad de cada uno. Es imposible estar seguro de si los objetos, que uno ha tomado en propiedad antes del surgimiento del conflicto que se busca dirimir, le pertenecen realmente a uno o si le corresponden a algún otro. Pues puede haber ocurrido que el otro haya decidido también poseer los mismos objetos. En tal caso no puede resolverse el conflicto entre ambas pretensiones de propiedad, ya que falta esta información relevante. Si alguna de las personas supiera con certeza que el otro se ha apropiado antes de los objetos, este conocimiento no serviría para resolver la cuestión, dado que este conocimiento se da en el foro interno de cada individuo, esto es, en el ámbito absolutamente subjetivo de la conciencia individual, $\mathrm{y}$, por esto mismo, es incomunicable e imposible de verificar objetivamente. Dependería, entonces, de la conciencia moral de cada individuo aceptar o no que los objetos que se quiere poseer ya pertenecen a un otro.

Lo mismo ocurrirá con los objetos que cada persona decida poseer a partir del momento en que ambas han entrado en relación intersubjetiva. Aun cuando las personas tengan la mejor voluntad de respetar la propiedad de la otra, no podrían decidir qué fines se ha propuesto la otra y qué objetos decidió utilizar para llevarlos a cabo. Es indispensable que esta información se exteriorice de alguna manera. En caso contrario, debería dejarse la solución de las pretensiones conflictivas librada al azar, esperando que ninguna persona desee o necesite los objetos que posee la otra. Pero incluso si se diera esta armonía casual, ella no garantizaría que se respete el principio del derecho y que la comunidad legal sea estable.

11 La incertidumbre e ignorancia de cada persona respecto de los fines que persigue la otra deben ser superadas porque, en caso contrario, la única opción que queda es la lucha de fuerzas entre las personas, lo que significa la muerte de toda relación legal. (cf. GA I, 3, 414-415 GNR) Por eso cada persona puede, a fin de formar una comunidad legal, obligar a la otra a declarar su posesión. Esto es, cada persona puede ser coaccionada para que diga expresamente qué parte del mundo sensible quiere poseer exclusivamente para llevar a cabo sus fines. Únicamente bajo esta condición es posible una relación intersubjetiva de acuerdo con el principio del derecho. ${ }^{4}$

Pero, si ambas personas declararan su voluntad de poseer el mismo objeto, esto no alcanzaría para resolver las pretensiones conflictivas, porque entonces ambas personas tendrían el mismo derecho. Este conflicto podría eventualmente solucionarse por medio de una negociación amigable, pero este camino no garantiza necesariamente que se llegue al equilibrio del derecho. Si una de las partes no quisiera ceder el objeto que 
pretende en favor de la otra, no podría ser coaccionada a ello, toda vez que todavía no se ha constituido una voluntad común.

Esto es, con la mera declaración de la voluntad de posesión quedan establecidas dos voluntades particulares, una frente a la otra, pero todavía no se ha constituido una voluntad que las unifique y que permita decidir el conflicto. (GA, I, 3, 416 GNR) Por eso, en caso de que no se llegue a un acuerdo voluntario, restará sólo la guerra entre las partes por la posesión del objeto. Por lo tanto, ambas partes deben delegar en un tercero la decisión respecto de las pretensiones conflictivas. Este acto supone que ambas partes deciden también abandonar el uso de la fuerza física y permitírselo sólo a este tercero. El tercero designado deberá no sólo resolver el litigio, sino también garantizar que su decisión será respetada y aplicada de modo estable en el futuro. Es decir, el tercero tendrá el poder de coacción sobre ambas partes a fin de cumplir con su rol de modo eficaz.

"Puesto que una tal guerra es, como toda guerra, ilegítima, para que [esta guerra] no se origine, tendrían que encargar a un tercero la resolución de su disputa [sc. la de las partes], cederle sin condicionamientos el derecho que ellos originariamente poseen a juzgar sobre el caso presente y la garantía de su resolución para el futuro. Por lo tanto [tendrían que] someter a él [sc. el tercero] su derecho a juzgar y su poder físico". (GA I, 3, 416-417 GNR) ${ }^{5}$

Él puede ejecutar la voluntad común a ambas, porque su voluntad particular no está involucrada en el conflicto.

Fichte denomina a esta instancia res publica o gemeines Wesen. (cf. GA I, 3, 417 GNR) La constitución de una voluntad común implica algo más que la mera declaración de la voluntad de posesión. La condición de la voluntad común es el reconocimiento recíproco del derecho de propiedad exclusiva de determinados objetos. ${ }^{6}$ Sólo el tercero designado puede reconocer su propiedad a cada una de las partes en conflicto. Cada persona debe excluirse a sí misma de la posesión del objeto, cuya propiedad reconoce al otro. Es decir, la propiedad tiene sentido sólo si la persona, a la par que se atribuye a sí misma una determinada cantidad de objetos del mundo sensible, renuncia a otra cantidad determinada. Si no lo hiciera, y pretendiera un derecho ilimitado a todos los objetos del mundo sensible, no podría coexistir en comunidad con otras personas.

La propiedad de la persona se determina por oposición a la de la otra, pero no por oposición a la propiedad de ella misma, porque esto sería absurdo. La persona no podría disputarse a sí misma la posesión de un objeto en el mundo sensible, porque se constituye como persona al entrar en la comunidad legal. Esto último es posible sólo si decide realizar sus fines en el mundo sensible por medio de la posesión exclusiva de determinados objetos. Por lo tanto ninguna persona puede aceptar ser desposeída de los objetos que necesariamente debe querer. La relación de determinación recíproca de la propiedad es en realidad un elemento constitutivo del proceso mismo de individuación. Según el $\S 4$ del GNR, el individuo se constituye como tal en la medida en que se contrapone a otros individuos y en el mismo acto se atribuye a sí mismo la autoría de sus acciones. Del mismo modo, la posesión (Besitz) deviene propiedad (Eigentum) en la medida en que es individuada, esto es, en que es distinguida de la propiedad de las otras personas por contraposición:

"Toda propiedad se funda en el reconocimiento recíproco y éste está condicionado por [una] declaración recíproca." (GA, I, 3, 417, GNR)

17 Como se dijo más arriba, la propiedad de la persona está garantizada en la medida en que los demás la reconocen y respetan. Por medio de la voluntad común que se expresa 
en el Estado, cada persona reconoce la propiedad de las demás. Pero queda aún otro factor que puede poner en peligro a la propiedad. Se trata de la amenaza de los ciudadanos de los demás Estados, que pueden esgrimir pretensiones conflictivas con respecto a la posesión de los objetos de los ciudadanos del primer Estado. De hecho la propiedad exclusiva de objetos del mundo sensible suele estar protegida dentro de los límites del propio Estado. Sin embargo, estas fronteras están en peligro siempre por la posibilidad de invasiones de los demás Estados o de guerras, o por el mero trato comercial que implica la posibilidad de que algunas cosas se puedan o se deban comprar o vender, etc.

Fichte encara este problema despejando en primer lugar la objeción de la invasión o la guerra por parte de otro Estado. No puede pensarse una comunidad legal si los individuos no respetan mutuamente sus esferas de acción, lo que excluye toda relación violenta, que tenga como finalidad el sometimiento o el exterminio del otro. Por lo tanto, desde el momento en que un Estado declara la guerra o invade otro, se trata de una relación que está fuera de todo estado de derecho. Sólo resta la lucha ciega de fuerzas entre los Estados, y carece de sentido intentar regular jurídicamente una guerra de este tipo.

El problema puede enfocarse mejor si se analiza el modo en que actúan los Estados cuando están en relaciones pacíficas. Aquí Fichte retoma su teoría del reconocimiento, desarrollada en los primeros parágrafos del GNR y en especial en los $\S \S 3$ y 4 . Fichte sostiene, en el $\S 3$ del GNR, que una persona reconoce a otra como tal no en virtud de un determinado concepto que tenga de la otra persona o de algún tipo de declaración verbal que haga, sino antes bien a partir de la constatación de que esa persona actúa respetando su esfera de acción libre. (cf. GA I, 3, 345 ss. y 356 GNR) De este modo, la persona está dándole a entender a la otra que considera que es un ser racional y que la exhorta a realizar una acción libre, en tanto que producto de su causalidad en el mundo sensible. (cf. GA I, 3, 340-348 GNR) Fichte traza una analogía con el reconocimiento de la propiedad de los ciudadanos de un Estado por los ciudadanos de otro Estado.

Desde el momento en que un Estado no está en guerra con otro Estado, está reconociendo a este último como un igual, por lo cual no intentará someterlo o invadirlo. Esto significa que este Estado le está dando a entender al otro que lo considera como un Estado capaz de soberanía y de autodeterminación. Por lo tanto, se compromete a respetar la propiedad de los ciudadanos del otro Estado, porque la función de este último consiste en garantizar los bienes y libertades que sus habitantes necesitan para actuar en el mundo sensible.

21 Esto se ve más claro si se considera el siguiente ejemplo dado por Fichte. Si un ciudadano llega al Estado en el que uno vive y declara no reconocer la propiedad de uno, robando un objeto que uno se ha apropiado exclusivamente para realizar sus fines, entonces este extranjero entrará necesariamente en contradicción consigo mismo, por las siguientes razones: en primer lugar, si este extranjero proviene de un Estado vecino y este Estado no está en guerra con el Estado de uno, en la medida en que este extranjero ha hecho uso de los objetos pertenecientes a la órbita de su Estado, ha reconocido a la voluntad común de este Estado vecino, esto es, ha reconocido también la propiedad de sus conciudadanos; en segundo lugar, también ha reconocido la propiedad mía y la de todos mis conciudadanos, porque ha reconocido la autoridad de la voluntad común que constituye su Estado, y esta ha reconocido la propiedad del Estado al que yo pertenezco (cf. GA I, 3, 418 GNR). Se trata de una especie de relación 
transitiva, que podría exponerse como sigue: si el ciudadano $\mathrm{A}$ ha reconocido la autoridad del Estado B, y este ha reconocido la del Estado C, entonces el ciudadano A también ha reconocido al Estado C. Lo mismo ocurriría si se tratara de un extranjero que llega a nuestro Estado desde un Estado muy lejano, que no tiene absolutamente ninguna relación con el nuestro, por lo cual no podemos decir que haya reconocido la propiedad de nuestros conciudadanos. Este extranjero ha recorrido necesariamente, para llegar a nuestro Estado, todos los Estados intermedios e incluso los mismos Estados vecinos, que han reconocido la propiedad de nuestro Estado. Pero ha debido entonces hacer uso de objetos o privilegios (por ejemplo los caminos) propios de estos Estados, los cuales sí reconocen nuestra propiedad. Por lo tanto, ha reconocido la propiedad de estos Estados e, indirectamente y de modo transitivo, la de nuestro Estado. ${ }^{8}$

Lo razonado hasta aquí se aplica a la propiedad reconocida recíprocamente por determinadas personas, esto es, a objetos del mundo sensible cuya apropiación exclusiva ha sido aceptada por los demás. Pero sigue siendo problemática la propiedad de los objetos que no han sido determinados por este contrato, que puede ser entendida como la propiedad de nadie (eine herrenlose Sache, res neutrius, cf. GA I, 3, 419) Este objeto, cuya propiedad nadie ha pretendido hasta ahora, puede ser poseído por un tercero, que no forme parte de la comunidad legal. Este caso quedaría totalmente fuera de la relación legal de propiedad y no constituiría, propiamente hablando, un problema.

Sin embargo, el intento de apropiación de esta herrenlose Sache por parte de alguno o de los miembros de esta comunidad, que ha reconocido la propiedad hasta el presente de los objetos ya distribuidos, pondría efectivamente en peligro la comunidad legal, hasta ahora establecida. Supongamos, pues, que una de las personas quisiera poseer uno de estos objetos que no han sido tomados en cuenta en el contrato de propiedad hasta el presente y que, por lo tanto, no son propiedad de nadie. Si otra persona pretendiera lo mismo, nos encontraríamos frente al mismo problema que había sido ya resuelto, porque cada persona expresaría la voluntad de poseer el mismo objeto, con el agravante de que a partir de lo ya acordado no podríamos dirimir este nuevo conflicto. Debería volver a recurrirse a la declaración y al reconocimiento subsiguiente cada vez, lo que quitaría estabilidad a la relación legal y postergaría permanentemente el problema de una regulación definitiva de la propiedad.

De este modo, estaría en peligro la comunidad legal misma, precariamente establecida con el primer reconocimiento de la propiedad entre los individuos, ya que no podría evitar el enfrentamiento de unos contra otros como medio para solucionar el conflicto. Por esta razón debe ampliarse la teoría de la propiedad desarrollada hasta aquí y tratar el problema no sólo del primer reconocimiento de la propiedad de las personas al momento del establecimiento de la comunidad legal, sino también el del reconocimiento de la propiedad de los demás en el futuro.

De lo que se trata, entonces, es del establecimiento de un mecanismo para resolver a futuro la apropiación de los objetos que han quedado indeterminados hasta ahora. Para resolver esta cuestión es necesario utilizar herramientas conceptuales nuevas. No se puede recurrir al derecho a la apropiación primera en el tiempo, esto es, al criterio, según el cual quien ha tomado el objeto antes que todos los demás es su propietario legítimo. Pues si un sujeto declara que ha poseído el objeto en primer lugar y el otro sujeto afirma lo mismo, no hay manera de resolver intersubjetivamente este conflicto, ya que la declaración de uno vale tanto como la del otro. Para llegar al reconocimiento 
intersubjetivo de la propiedad en litigio no debe tener lugar primero la declaración de la propiedad y luego su toma efectiva de posesión, sino que más bien ambas deben ocurrir simultáneamente.

Por lo tanto, cuando un individuo conoce el objeto, debe simultáneamente poder comprender que este pertenece a un sujeto en particular. Para que se dé esta simultaneidad el objeto debe estar acompañado de una determinada marca identificadora que lo remita a su poseedor. Esta marca debe ser convenida de antemano por los sujetos, de modo tal que se evite toda discusión respecto de quién ha tomado el objeto en primer lugar. Un ejemplo de Fichte puede ilustrar esto claramente: si las personas se distribuyen parcelas de tierra y convienen que todo lo que crezca en ellas le pertenecerá a su propietario, resolverán así las pretensiones de propiedad a futuro simplemente con cercar el terreno. Esto es, con sólo conocer la ubicación del objeto en cuestión. Por ejemplo: se podrá determinar que tal o cual vegetal creció en el terreno adjudicado a cierta persona y que, de acuerdo con lo convenido, pertenece a ésta. De este modo, se asegura un criterio que dota de estabilidad a la propiedad de los miembros de la comunidad legal, sin tener que recurrir a la declaración y al reconocimiento en cada caso. (cf. GA, I, 3, 421 GNR)

Dado que lo fundado no puede ir más allá de su fundamento y que la declaración de posesión es el fundamento de la propiedad, si una persona declara el abandono de la propiedad (derelictio domini) de algún objeto en particular, se considerará que este objeto determinado ya no es más suyo. De no mediar esta declaración, se considerará que la persona sigue en posesión del objeto. Pero puede ocurrir que el propietario descuide los signos que identifican al objeto de su posesión y que estos terminen desapareciendo o volviéndose irreconocibles (por ejemplo que no mantenga su campo alambrado). Siguiendo el principio fichteano de que lo fundado no puede ir más allá de su fundamento, en este caso se considera a la propiedad como abandonada, toda vez que la posesión se determina por los signos que la indican. De lo cual se sigue que:

"El signo no es, por lo tanto, nunca algo superfluo, sino el fundamento necesariamente permanente del derecho; y si el propietario lo retira o deja que se arruine hasta desaparecer, debe ser, entonces, considerado como un propietario que ha abandonado su derecho de propiedad." (GA I, 3, 422, GNR) ${ }^{9}$

El equilibrio del derecho consiste en la determinación de esferas iguales de acción para todas las personas afectadas. Como toda determinación, requiere de realidad y concreción, lo que en este caso se logra gracias al mutuo reconocimiento de la propiedad según los mecanismos analizados más arriba. De este modo, se despeja la incertidumbre respecto de la propiedad de los objetos en disputa y puede garantizarse un derecho real a una cantidad de objetos del mundo sensible para cada persona. Así se ha dado el paso desde el mero conocimiento del otro como un ser racional hasta la aplicación completa del principio del derecho a cada caso en particular por medio del contrato de propiedad.

\section{El derecho de coacción}

Ahora bien, la demostración del equilibrio del derecho permanece incompleta, dado que falta determinar su condición de posibilidad. Esto es, no basta con que las personas acuerden la mutua determinación de sus derechos de propiedad, tanto en lo atinente a las pretensiones conflictivas presentes como a las que surjan a futuro. Si el contrato de 
propiedad debe tener validez para todo el futuro, debe presuponerse una condición fundamental como dada de antemano, consistente en que los sujetos se sometan de hecho a la ley jurídica siempre y sin excepciones. (cf. GA I, 3, 423-424, GNR) En caso contrario, la comunidad legal no tendría la estabilidad y la permanencia que su concepto exige. Pero esta condición, que se da por supuesta, en realidad debe ser demostrada. Por esto debemos ocuparnos, en lo que sigue, de este problema a fin de completar la fundamentación del equilibrio del derecho.

La condición del cumplimiento efectivo y estable del derecho de propiedad es entonces que los contratantes cumplan la ley sin excepciones. Pero además es necesario que cada uno pueda saber que el otro ha tomado como fin de sus acciones el cumplimiento de la ley. Si consideramos la motivación de las personas a la hora de reconocerse mutuamente la propiedad, puede ocurrir que no pacten en pro de la convivencia legal misma, sino más bien motivadas por el miedo a ser despojadas por la otra persona. Pero este móvil no otorgaría estabilidad a la relación legal, dado que podría ocurrir que, una vez que reine la paz entre los contratantes y cada uno tenga su propiedad garantizada, uno de los dos crea que el otro está en situación de debilidad y decida entonces quebrar el pacto $y$ atacarlo. ${ }^{10}$

31 Toda vez que las personas no tienen manera de saber si la otra cumplirá o no con lo pactado, tampoco tienen la obligación de someterse a la ley, porque no pueden poner en riesgo su libertad en virtud de su derecho originario e inalienable a actuar libremente en el mundo sensible. Ahora bien, parecería que cada contratante debería confiar en la buena fe del otro a fin de prever el cumplimiento futuro de lo acordado. Este es el sentido de la fórmula de Fichte:

"La posibilidad de la relación de derecho entre personas en el ámbito del derecho natural está condicionada por la confianza y fe recíproca." (GA I, 3, 424, GNR) ${ }^{11}$

Pero la confianza y la buena fe caen fuera del ámbito del derecho externo, porque pertenecen al foro interno de la persona, es decir, al ámbito de su conciencia moral. Esto es, nadie puede ser obligado a tener determinado sentimiento o pensamiento, porque sólo la persona misma tiene acceso a la privacidad de su propia conciencia. Por estas razones, no puede establecerse todavía el derecho de propiedad y no se puede concretizar la comunidad legal. En este contexto, los individuos están justificados al entablar una lucha a muerte entre sí por los objetos del mundo sensible que necesitan apropiarse para la consecución de sus fines, o sólo pueden esperar que los otros lo hagan y colocarse así en una actitud defensiva. Por lo tanto, reina aquí la desconfianza mutua entre los miembros de la comunidad legal.

Fichte invoca entonces la distinción entre el derecho y la moral, según la cual el recurso a la moral es insuficiente para dar estabilidad a la relación legal. Por lo tanto, debe diseñarse un sistema legal que no fundamente su estabilidad en la confianza en la buena disposición de las personas a cumplir con la ley. Fichte considera que la confianza y la buena fe (Treue und Glauben) son muy frágiles e inestables, dado que suponen una voluntad inquebrantable en el cumplimiento de la ley, que difícilmente pueda esperarse tanto de nosotros cuanto de los demás. Aunque reinaran la buena fe $\mathrm{y}$ la confianza, basta con una sola transgresión del contrato de propiedad por parte de alguna de las personas, para que la confianza mutua se pierda irremediablemente. La otra persona tomará precauciones para defenderse en caso de volver a sufrir un atentado contra su propiedad por parte de la otra, y ésta se verá obligada a hacer también lo propio. Con esto, la mutua desconfianza generará una escalada simétrica de 
violencia y una hostilidad creciente, que posiblemente termine en un enfrentamiento de todos contra todos. ${ }^{12}$

Pero cada miembro de la comunidad legal no está interesado necesariamente en que el otro desarrolle las disposiciones morales de la buena fe y la confianza, sino sólo en que se comporte como si tuviera una voluntad firme e inquebrantable de cumplir con la ley, aunque de hecho no la tenga. Esto es, cada uno espera del otro simplemente que cumpla la ley sin hacer excepciones. Como consecuencia, la moralidad queda relegada al foro interno de la conciencia, y no puede ser objeto del derecho, que se ocupa sólo del efecto del obrar de las personas en el mundo sensible. Dicho de otro modo:

"Todo individuo tiene solamente derecho a exigir la legalidad [en las acciones] del otro, de ningún modo que éste actúe moralmente" (GA I, 3, 425, GNR) ${ }^{13}$

El dispositivo destinado a coaccionar a las personas para garantizar que cumplan con la ley debe dirigirse a su voluntad misma, a fin de forzarla a que se autodetermine a querer los fines que estén de acuerdo con la ley, tanto en el caso de que tenga una buena como una mala voluntad. Fichte descarta de plano la necesidad de idear un mecanismo coactivo para actuar sobre una voluntad buena, porque esta consiste justamente en querer la ley por sí misma. La voluntad buena hace suyo el deber de cumplir con la ley y, por eso, no debe ser determinada en orden a querer este principio. (cf. GA I, 3, 426)

El punto de partida para esta teoría es más bien aquella voluntad que no quiere cumplir con la ley. Fichte idea un dispositivo ordenado a la coacción de la voluntad mala, esto es, de aquella que atenta intencionadamente contra el derecho, procurando apropiarse de la libertad y los objetos que le pertenecen al otro. El razonamiento jurídico prohíbe suponer que, quien tiene una voluntad mala, puede llegar a regenerarse moralmente. Por lo tanto, el dispositivo legal debe ser construido de modo tal que se logre que, cada vez que la voluntad busque un fin ilegal, se produzca el efecto exactamente contrario. Si la voluntad mala desea un efecto pero siempre resulta el contrario, luego de un tiempo dejará de buscar el fin nocivo. ${ }^{14}$ Dicho de otro modo, se anulará a sí misma en cuanto voluntad mala.

"Toda voluntad que se oponga al derecho devendría el fundamento de su propia aniquilación, así como la voluntad sin más es el último fundamento de sí misma" $(\mathrm{GA}, \mathrm{I}, 3,426, \mathrm{GNR})^{15}$

37 La propuesta de Fichte consiste entonces en que la ley esté estructurada de modo tal que, si la voluntad individual del criminal persigue incrementar su propiedad a costa de los demás, finalmente pierda toda su propiedad a causa de su mismo delito. Por lo tanto, el delincuente deberá calcular las consecuencias de su acción desde su autointerés, y concluirá que la realización del crimen le traerá más desventajas que ventajas. De este modo, el ciudadano cumplirá con la ley y contribuirá con el bien común por mero egoísmo racional. Pero sólo una ley, una determinación conforme a reglas, puede modificar a una voluntad mala de modo permanente. Por esta razón Fichte propone el derecho de coacción.

“Así pues, si pudiera ser ejecutado un dispositivo, que funcione con necesidad mecánica, por medio del cual resultara de cada acción ilícita lo contrario de su objetivo, la voluntad sería, entonces, constreñida - por medio de un tal dispositivo a querer solo aquello conforme a derecho. Al ser conducida la voluntad mala y que codicia bienes ajenos justamente por medio de su codicia no conforme a derecho hacia este mismo objetivo, la seguridad sería, por medio de este dispositivo, reestablecida, luego de que la confianza y la buena fe [entre las partes] se haya 
perdido, y la buena voluntad se volvería prescindible [como condición] para la realización externa del derecho. Un dispositivo como el descrito significa una ley de coacción." (GA, I, 3, 427, GNR) ${ }^{16}$

\section{El problema de la implementación del derecho de coacción}

Ambos contratantes acuerdan garantizarse recíprocamente la seguridad de su propiedad pero, a falta de la buena fe y la confianza en el otro, deben acordar también que el posible agresor sea sometido al derecho de coacción. Cada persona acordará necesariamente la aplicación del derecho de coacción, dado que quien quiere el fin quiere los medios necesarios para ello, y este derecho es el único modo de garantizar la conservación de la propiedad, según lo argumentado en el § 14 del GNR y explicado en el punto anterior del presente trabajo. Es decir, cada persona consentirá en que se ejerza una fuerza tal sobre el agresor que, a causa de la acción por medio de la cual busca conservar e incrementar su propiedad, este derecho resulte lesionado de igual modo.

Debe tratarse de una fuerza o potencia que el agresor no pueda resistir y que se aplique sobre él inevitablemente, para que el agresor cuente con ella cuando planifique su delito. De acuerdo con este mecanismo, la voluntad del agresor, ya se trate de un agresor intencional o de uno negligente, será determinada en orden al fin de respetar la propiedad de cada contratante. La potencia que se aplica sobre al agresor resulta de la voluntad común a los contratantes $\mathrm{y}$, por lo tanto, no es una mera fuerza mecánica, sino más bien una fuerza determinada según un concepto elaborado por todos los contratantes, esto es, un concepto fruto de su libertad. Se trata del concepto de los límites que cada contratante debe poner a su libertad, a fin de garantizar la libertad de los demás. En cuanto a la composición de esta fuerza, resulta de la unión de la potencia de los contratantes en una sola, por lo cual es siempre más potente que la fuerza de cada contratante tomada por separado.

Los contratantes deberán resolver otro problema fundamental, para poder aplicar el derecho de coacción, que es el de la designación del depositario de este poder superior al de los demás (Übermacht). En primer lugar, el contrato no puede distribuir el poder entre los individuos de modo tal, que ambos tengan la misma fuerza después de cometida la transgresión. Veamos qué ocurriría si el agresor tuviera el mismo poder que antes de la agresión. En este caso, el agresor podría aplicar la coacción contra sí mismo, a fin de determinar su voluntad. Se trataría de un contrato contradictorio y, por lo tanto, nulo, dado que, si el agresor se resistiera a la acción transgresora, entonces ésta nunca tendría lugar. Lo cual significa que la aplicación de un derecho de coacción carecería de sentido.

41 Entonces el contrato podría contener una cláusula que dijera que todas las personas pueden aplicar la coacción sobre el posible agresor, pero que el agresor no debe nunca ofrecer resistencia a esta coacción. Pero esto tampoco sería coherente, toda vez que la necesidad de la coacción de una voluntad surge de su falta de conformidad con la ley. Si esta voluntad no ofreciera resistencia alguna a la misma, entonces estaría determinada conforme a la ley, lo cual volvería inútil la aplicación de la coacción sobre ella. (cf. GA, I, 3, $430 \mathrm{GNR}$ ) Si se atribuyera a las dos personas la misma fuerza para coaccionar, independientemente de quién fuera la agresora, entonces la persona agredida debería 
confiar y ponerse en las manos de quien la ha atacado, lo cual no puede pedírsele, toda vez que ya ha perdido la confianza en él.

Ambas personas podrían acordar entonces entregar todo el poder en manos del agredido, para hacer justicia al agresor. Si cada uno consiente en entregar toda su libertad en manos de aquél a quien ofenda, entonces se abstendría de agredirlo. Sin embargo, esta solución también es contradictoria. Si el agresor dejara su libertad en manos del agredido, no tendría garantía alguna de que la víctima use este poder excesivo que le ha sido confiado para, a su vez, atentar también contra la libertad y la propiedad del agresor que es castigado. Si se intenta contrarrestar esta consecuencia por medio de un mecanismo que permita al agresor juzgar el modo en que el agredido lo coacciona, esto conllevaría que el agredido se ponga en manos del agresor.

Esto es, el agredido debería confiar en el buen juicio del agresor, y partir de su buena fe. Pero no pueden recuperarse la confianza y la buena fe luego de la agresión sufrida, según el §14 (GA I, 3, 425, GNR). Por lo tanto, las personas se obligarían mutuamente a hacer algo que escapa a sus posibilidades, lo cual es contradictorio. No puede acordarse, entonces, que el agredido y el agresor conserven el mismo poder que tenían antes del quiebre del contrato por parte de uno de los dos. Más bien parecería que debe otorgarse más poder al agredido que al agresor, toda vez que el mecanismo de coacción consiste en que el agresor pierda la seguridad de su propiedad como consecuencia de sus actos. Pero Fichte rechaza esta posibilidad, argumentando que el agredido podría utilizar el poder superior que se ha puesto en sus manos para transgredir la ley. Entonces la facultad de coaccionar debe ser delegada en alguien que no sea ni el agresor ni el agredido, es decir, en un tercero. ${ }^{17}$

A fin de que el contrato de propiedad sea efectivo, los contratantes deben unificar su voluntad según el contenido del derecho de coacción y delegar toda su fuerza en un tercero, que garantizará la imparcialidad en la aplicación del derecho. ${ }^{18}$ Este tipo de organización, que Fichte denomina res publica, permite la realización estable de la comunidad jurídica. El derecho natural a la causalidad libre en el mundo sensible está atado al establecimiento de un determinado derecho positivo en el seno de una res publica. La teoría del derecho natural entonces no puede ser pensada sin el diseño institucional de un Estado justo y efectivo. En caso contrario, el derecho natural no sería más que una mera abstracción vacía y sin anclaje alguno en la realidad. El supuesto motivacional del egoísmo universal es la garantía de la estabilidad de la comunidad legal.

A partir de aquí Fichte desarrolla una teoría del contrato social, del derecho de propiedad, del justo castigo, del matrimonio y del problema de la guerra. Tanto en el GNR como en el Estado comercial cerrado (1800) Fichte sostiene que el Estado debe planificar centralmente la economía, tanto la producción como la distribución de mercancías, para permitir a los ciudadanos que vivan de su trabajo. Esta propuesta, que ha ejercido influencia sobre el socialismo, ${ }^{19}$ puede ser interpretada adecuadamente sólo si se tiene en cuenta la idea del equilibrio del derecho y la necesidad de un derecho de coacción, tal como se las ha desarrollado en este trabajo. Ha sido mi intención hacer un aporte en esta dirección. 


\section{NOTAS}

1. Las traducciones de los pasajes citados de este texto en el presente trabajo son de E. Acosta.

2. G. W. F. Hegel „Über die wissenschaftlichen Behandlungsarten des Naturrechts, seine Stelle in der praktischen Philosophie und sein Verhältnis zu den positiven Rechtswissenschaften“; en: Kritisches Journal der Philosophie, II, 2, (Noviembre/Diciembre, 1802), y II, 3 (Mayo/junio ,1803).

3. "Wenn, wie es bei Deduction des Urrechts geschieht, eine Person in der Sinnenwelt isolirt gedacht wird, so hat sie so lange, als sie keine Person ausser ihr kennt, das Recht, ihre Freiheit so weit auszudehnen, als sie kann und will, und, wenn es ihr beliebt, die ganze Sinnenwelt für sich in Besitz zu nehmen. Ihr Recht ist wirklich (wenn das Urrecht nur überhaupt ein wirkliches Recht seyn könnte) unendlich, denn die Bedingung, unter der dasselbe beschränkt seyn müsste, fällt weg."

4. H.-J. VERWEYEN, Recht und Sittlichkeit in J. G. Fichtes Gesellschaftslehre. München-Freiburg 1975: 103 .

5. "Da nun ein solcher Krieg, so wie aller Krieg, absolut widerrechtlich ist, müssen sie, damit er nicht entstehe, die Entscheidung ihres Streites einem Dritten übergeben, ihm ohne Vorbehalt ihr Rechtsurtheil, über den gegenwärtigen Fall, und die Garantie seiner Entscheidung, für die Zukunft, überlassen, also ihr Recht zu urtheilen, und ihre physische Macht ihm unterwerfen“".

6. R. P. HORSTMANN “Theorie des Urrechts (§§ 8-12)“, en: J.-Ch. Merle (ed.), Johann Gottlieb Fichte. Grundlage des Naturrechts, Berlin 2001: 122.

7. "Alles Eigenthum gründet sich auf wechselseitige Anerkennung, und diese ist bedingt durch gegenseitige Declaration."

8. Si bien las consideraciones de Fichte sobre la propiedad en el § 12 están orientadas meramente a la fundamentación del derecho de coacción, son propedéuticas para la teoría de la propiedad de la segunda parte del GNR. (véase GA I, 4, 20-58 GNR)

9. „Das Zeichen ist also nie etwas Ueberflüssiges, sondern der nothwendig fortdauernde Rechtsgrund; und wenn der Eigenthümer dasselbe wegnimmt, oder es zu Grunde gehen lässt, so ist er anzusehen als ein solcher, der sein Eigenthumsrecht aufgegeben hat."

10. Cfr M. KaufmanN, "Zwangsrecht (§§ 13-16)“, en: J.-Ch. Merle (ed.) Johann Gottlieb Fichte. Grundlage des Naturrechts. Berlin 2001:.129.

11. "Die Möglichkeit des Rechtsverhältnisses zwischen Personen auf dem Gebiete des Naturrechts ist durch gegenseitige Treue und Glauben bedingt."

12. Cf. R. ZACZYK, Das Strafrecht in der Rechtslehre J. G. Fichtes. Berlin 1981: 71.

13. "Jeder hat nur auf die Legalität des Anderen, keinesweges auf seine Moralität Anspruch.“

14. Acerca del efecto disuasorio del derecho de coacción véase L. FONNESU "Die Aufhebung des Staates bei Fichte“, en: F-S 11, 1997: 94.

15. "Jeder unrechtmässige Wille würde der Grund seiner eigenen Vernichtung, so wie der Wille überhaupt der letzte Grund seiner selbst ist.“

16. "Wenn demnach eine mit mechanischer Nothwendigkeit wirkende Veranstaltung getroffen werden könnte, durch welche aus jeder rechtswidrigen Handlung das Gegentheil ihres Zwecks erfolgte, so würde durch eine solche Veranstaltung der Wille genöthigt, nur das Rechtmässige zu wollen; durch diese Anstalt würde, nach verlorener Treue und Glauben, die Sicherheit wiederhergestellt, und der gute Wille für die äussere Realisation des Rechts entbehrlich gemacht, indem der böse und nach fremden Sachen begierige Wille, gerade durch seine eigene unrechtmässige Begier, zu dem gleichen Zwecke geleitet würde. Eine Veranstaltung, wie die beschriebene, heisst ein Zwangsgesetz." Véase con respecto a esto M. KAUFMANN, op. cit. 132-133. 17. Cf. R. ZACZYK op. cit. 72. 
18. Cf. H. VERWEYEN op. cit. 104.

19. La influencia de la teoría fichteana sobre algunos socialistas es evidente en casos como el de Ferdinand Lasalle, Marianne Weber y Franz Mehring.

\section{RESÚMENES}

In this paper I aim to reconstruct the argumentation, which Fichte elaborates in his Foundations of Natural Right (1796/1797), in order to give the foundations of the right to coercion. I intend to contribute to the comprehension of the Fichtean theory of the state, wich had a great influence upon the socialist thought.

ÍNDICE

Keywords: right, coercion, state, equilibrium

\section{AUTOR}

\section{HÉCTOR ARRESE IGOR}

La Plata, Argentina 